\title{
IMPULSIVE FLARE PLASMA ENERGIZATION IN THE LIGHT OF YOHKOH DISCOVERIES
}

\author{
YURII M. VOITENKO \\ Department of Space Plasma Physics, Main Astronomical \\ Observatory, Holosiiv, Kyiv-22, 252650, Ukraine
}

Flare scenario, based on Yohkoh observations and magnetic reconnection (MR) hypotesis, has been elaborated recently (Tsuneta, 1996; Shibata, 1997). Using theoretical prediction that the fast MR results in a high-speed outflows from reconnection site, the reconnection downflow has been supposed to collide with the top of underlying magnetic loop, producing superhot plasma and high-energy particles.

We take into account another part of the reconnection outflow, diverting along field lines close to separatrix, and forming so-called separatrix jets (Strachan and Priest, 1994). These sepatatrix jets set up warm beams of $\geq 0.1 \mathrm{MeV}$ protons (PBs), streaming down along just-reconnected field lines through steady underlying plasma (Voitenko, 1996a;b). Note that there are many observations indicating that during initial phase the bulk flare energy is carried by $\geq 0.1 \mathrm{MeV}$ proton beams (Simnett, 1995). The principal significance for the solar flares theory has a question of the further transformation of PB kinetic energy in flaring loops (FLs), overlying well-known SXR loops.

We study excitation of kinetic Alfvén waves (KAWs) by PB, keeping effects of induced by PB large-scale electric field $\mathbf{E}_{\mathbf{0}} \| \mathbf{B}_{\mathbf{0}}$ along the legs of flaring loop (Voitenko, 1996a). Taking into account high growth rate $\left(\sim 10^{4} \mathrm{~s}^{-1}\right)$, short relaxation distance $\left(\sim 10^{6} \mathrm{~cm}\right)$, and energy flux partitition between waves and PB after relaxation $\left(P_{K A W} / P_{P B} \sim 1\right)$, we conclude that PB-driven KAW instability is an efficient mechanism of flare energy conversion in FL. The quasi-linear spectra of excited waves $\sim k_{\perp}^{2}$ in longwavelength domain, and $\sim k_{\perp}$ in short-wavelength domain. Spectral energy concentration at largest wavenumbers below upper cut-off, where nonlinear three-wave interaction is strongest, give rise to nonlinear modification of the quasilinear spectra and to the formation of turbulent spectra $\sim k_{\perp}^{-5}$ in 
long-wavelength domain, and $\sim k_{\perp}^{-3}$ in short-wavelength domain.

Flare plasma energization by KAW turbulence may be responsible for various observed phenomena with rise time and time delays of about 5-10 s, corresponding to $\mathrm{PB} / \mathrm{KAW}$ flux propagation time. Impulsive heating of plasma near injection site by a strong KAW flux results in time dependence of electron temperature in the upper part of FL legs (Voitenko, 1996b):

$$
T_{e(7)}=\left(1.4 \times 10^{4} B_{i n(2)} t+T_{0}^{5 / 2}\right)^{2 / 5},
$$

where $T_{0(7)}$ is initial temperature, and subscripts in () denote the order (e.g., $T_{e(7)}=T_{e} / 10^{7}$, etc.).

1992 Jan. 13 flare (Masuda et al., 1995). Taking plasma number density $n_{0(9)}=2.5$ in legs and $n_{0(9)}=10$ at the top, FL half-length $L_{(9)}=2$, $n_{b(9)}\left(\approx n_{i n(9)}\right)=1, B_{0(2)}\left(\approx B_{i n(2)}\right)=0.57, T_{0(7)}=0.6$, and PB injection time $t_{b}=3 \mathrm{~s}$, we obtain high instability growth rate, $\gamma_{k}=3 \times 10^{4} \mathrm{~s}^{-1}$, short relaxation distance $7 \times 10^{5} \mathrm{~cm}$, temperature of heated region $T_{e}=$ $6.7 \times 10^{7}$, spreading velocity $\geq 4 \times 10^{8} \mathrm{~cm} \mathrm{~s}^{-1}$ and flux of escaping $(>20$ $\mathrm{keV}$ ) electrons $10^{17} \mathrm{el} . \mathrm{cm}^{-2} \mathrm{~s}^{-1}$. Hence the fast plasma heating by KAWs provides temperature and flux of escaping electrons, enough to produce observed (thermal synchrotron) microwave emission from the loop leg, and HXR bremsstrahlung from footpoints and loop top.

1992 Feb. 21 LDE flare (Tsuneta, 1996). In inflow region $n_{i n(9)}=$ $1, T_{e(7)}=0.5 ;$ in FL legs $n_{0(9)}=5, T_{e(7)}=1.3$; heat conduction loss from legs $P_{t h(9)}=1$. Then PB number density $n_{b(9)} \approx 1$ and (super-Alfvén) velocity $V_{0 b} / V_{A} \approx 2.2$. Assuming that the heating of FL legs by KAW flux is balanced by $P_{t h}$, we get reasonable estimation for flare energy flux $P_{f l(9)} \approx P_{0 b(9)}=2.5$ and reconnecting field $B_{0(2)}=0.2$.

Acknowledgements. Useful discussions of flare models with K. Shibata and S. Tsuneta are gratefully acknowledged. This work was supported in part by grant 2.4/1003 of the Ukrainian Fund for Fundamental Research; the presentation at the IAU Symposium No. 188 became possible due to IAU travel grant.

\section{References}

Masuda, S., Kosugi, T., Hara, H., Sakao, T., Shibata, K., and Tsuneta, S.: 1995, P.A.S.J. 47,677

Shibata, K.: 1997, in T. Watanabe, T. Kosugi, and A. C. Sterling (eds) Proc. Yohkoh 5th Anniversary Symposium, in press.

Simnett, G. M.: 1995, Space Sci. Rev. 73, 387.

Strachan, N. R. and Priest, E. R.: 1994, Geophys. Astrophys. Fluid Dynamics 74, 245-274

Tsuneta, S.: 1996, Astrophys. J. 456, 840.

Voitenko, Yu. M.: 1996a, Solar Phys. 168, 219.

Voitenko, Yu. M.: 1996b, ASP Conference Series 111, 312. 